\title{
Tumor sólido pseudopapilar do pâncreas
}

Case Repomt

\section{Solid pseudopapillary tumor of the pancreas}

Ivan Maluf Junior ${ }^{1}$

Mariana Ribas Zahdi ${ }^{1}$

Luciane Valdez ${ }^{2}$

\section{Resumo}

O tumor sólido pseudopapilar de pâncreas é uma neoplasia rara, que acomete usualmente mulheres jovens na segunda ou terceira década de vida. Foi descrito pela primeira vez em 1959. É considerada uma doença com baixo potencial de malignidade e é encontrado na maioria dos casos em adolescentes do sexo feminino, sugerindo a associação de fatores hormonais em sua patogênese. Metástases são raras e a ressecção completa do tumor é geralmente curativa. O caso descrito é sobre uma paciente de 13 anos. Os aspectos clínicos envolvidos neste caso foram atípicos pelo fato do surgimento dos sintomas associados a trauma direto com aumento de volume abdominal. O tumor pode acometer qualquer área do pâncreas, porém, na maioria das séries, acomete, preferencialmente, o corpo e a cauda pancreática. A ressecção do tumor, quando possível, é curativa na maioria dos casos, porém recidivas locais podem ocorrer. A taxa de sobrevida mesmo após metástase hepática é alta pela natureza benigna do tumor. Conclusão: O tratamento cirúrgico por meio de ressecção tumoral sem esvaziamento ganglionar ou ressecção de órgãos adjacentes é recomendável pela literatura internacional proporcionando excelente prognóstico para estes pacientes.

\begin{abstract}
Introduction: Solid pseudopapillary tumors of the pancreas (SPTs) are uncommon tumors usually affecting young women in their twenties and thirties, suggesting hormonal factors to be involved in their pathogenesis. It was first described by Frantz in 1959. The vast majority of SPTs are indolent neoplasms. Metastases are rare and resection of the tumor when possible is curative in most cases. Case report: We report a case of a 13-year-old white girl. The clinical aspects of this case were atypical due to the appearance of symptoms associated with direct trauma and increase of the abdominal volume. Discussion: The body and tail of the pancreas are more frequently affected. The survival rate even after liver metastasis is good. Conclusion: Surgery is the mainstay of treatment. Complete resection of the tumor is usually achieved. Extensive lymphatic dissection or more radical local approaches are not indicated by most studies.
\end{abstract}

Palavras-chave: Neoplasias Pancreáticas.

Key Words: Pancreatic Neoplasms

${ }^{1}$ Acadêmicos do $6^{\circ}$ ano, Curso de Medicina, Universidade Positivo, Curitiba, Paraná, Brasil.

${ }^{2}$ Médica oncologista infantil e pediatra, Serviço de Oncologia e Hematologia, Hospital Infantil Pequeno Príncipe, Curitiba, Paraná, Brasil.

135 Rev Bras Med Fam e Com

Rio de Janeiro, v.4, n¹4, jul /set 2008 


\section{Introdução}

O tumor sólido pseudopapilar do pâncreas (TSPP), também conhecido como tumor de Frantz é uma neoplasia rara do pâncreas, ocorrendo em aproximadamente 0,17\% $2,7 \%$ dos tumores não-endócrinos do pâncreas. Foi descrito pela primeira vez em 1959. Embora rara, é uma neoplasia que tem sido cada vez mais descrita na literatura. Até o momento, existem apenas 452 casos citados na literatura ${ }^{1,2,3}$.

No Brasil, a maior casuística em uma única instituição foi apresentada pelo Serviço de Cirurgia de Vias Biliares e Pâncreas do Hospital das Clínicas da Faculdade de Medicina da Universidade de São Paulo, com 14 casos submemetidos à cirurgia ${ }^{1}$.

É encontrado na maioria dos casos em adolescentes do sexo feminino sugerindo a associação de fatores hormonais em sua patogênese ${ }^{3}$.

É considerado uma neoplasia com pequeno grau de malignidade, desde que passível de ressecção cirúrgica completa, o que é possível na maioria dos casos ${ }^{3}$.

Os achados clínicos são vagos e podem incluir dor abdominal leve e saciedade precoce como principal manifestação além de massa palpável em quadrante superior esquerdo ${ }^{1,2,3}$.

A evolução do tumor é de crescimento lento, com tendência ao sangramento intratumoral e necrose. Por essa razão pode alcançar grandes volumes e apresenta-se clinicamente na forma de massa abdominal ${ }^{3}$.

A despeito disso, é rara a invasão de estruturas vasculares ou biliares, o que torna a ressecção possível na maioria dos pacientes ${ }^{2}$.

tumor pode acometer qualquer área do pâncreas, porém, na maioria das vezes acomete, preferencialmente, o corpo e a cauda pancreática. Também foram descritos tumores de formas multicêntricas com aparecimento em tecido pancreático ectópico. Histologicamente se caracteriza por apresentar uma mescla de áreas sólidas com pseudocistos, estruturas pseudopapilares e hemorrágicas $^{2}$.

O ultrassom e a tomografia são os exames mais apropriados para fazer investigação diagnóstica. Geralmente, observa-se uma massa pancreática bem delimitada, encapsulada, com calcificações e padrões sólidos e císticos dependendo do grau de hemorragia ${ }^{2}$.

O conhecimento dos aspectos epidemiológicos e clínicos desse tipo de tumor pode levar à suspeita do diagnóstico no pré-operatório, o que, sem dúvida, pode permitir abordagem cirúrgica mais radical que tenha por objetivo a ressecção por completo de lesão. O prognóstico após ressecção tumoral é excelente com índice de sobrevivência de 90\% e recorrência de apenas 10\%. Metástases hepáticas são raras $(7 \%)$ e acometem na maioria dos casos pacientes de idade mais avançada ${ }^{3}$.

\section{Objetivo}

Este caso foi selecionado por apresentar um quadro clínico atípico de tumor abdominal, com diagnóstico de neoplasia rara e poucos casos relatados na literatura.

\section{Relato de caso}

Paciente M.C.F., 13 anos, sexo feminino, natural de Salto da Lontra (PR). Apresentava quadro clínico de dor abdominal localizada, episódios esporádicos de náuseas e vômitos e massa abdominal em hipocôndrio esquerdo.

No dia 04 de janeiro de 2007, a paciente havia sofrido trauma abdominal direto e iniciou quadro de vômitos. Ficou em observação por aproximadamente quatro horas na cidade de origem. Não houve melhora. Foi realizada ultrassonografia abdominal, e a paciente foi encaminhada ao Hospital Infantil Pequeno Príncipe no dia 19 de janeiro de 2007.

Ao exame físico, a paciente apresentava-se em bom estado geral, calma e ativa. Peso de $35 \mathrm{~kg}$, frequência cardíaca de 88 bpm e frequência respiratória de $20 \mathrm{rpm}$. Abdômen flácido, com massa palpável $4 \mathrm{~cm}$ abaixo do rebordo costal esquerdo, fígado impalpável.

O exame de ultrassonografia realizado no dia 18 de janeiro de 2007 demonstrou massa nodulariforme, com contornos predominantes definidos e ecogenicidade heterogênea, medindo cerca de 8x7,9x6,9 cm em hipocôndrio esquerdo, medial ao baço e superior ao rim. 
A paciente foi internada. Foi solicitada Tomografia Computadorizada posteriormente, a qual demonstrou região cefálica e torácica sem alterações. Abdômen com lesão expansiva, nodulariforme, heterogênea, predominantemente hipodensa, localizada em hipocôndrio esquerdo, medialmente ao baço e anteriormente ao rim esquerdo. A extensão do tumor era de 9x7, 9x7,5 cm e apresentava impregnação heterogênea após injeção endovenosa do contraste, predominando em região periférica.

O hemograma apresentava: leucócitos 20300/ ml, basófilos zero, eosinófilo $2 \%$, bastão $1 \%$, segmentados $61 \%$, monócitos $19 \%$, linfócitos $17 \%$, plasmócitos zero. Série vermelha normal. Amilase sérica $31 \mathrm{UA} / \mathrm{ml}$, creatinina $0,6 \mathrm{mg} /$ dL. Dosagem de Na e K dentro da normalidade. TGO de 126 U/L e TGP de 18 U/L. Gama GT 11UI/L, LDH 537 U/L e Fosfatase alcalina de $112 \mathrm{U} / \mathrm{dL}$.

Exames de urina sem alterações. Foi realizada cirurgia para retirada de massa abdominal no dia 23 de janeiro de 2007, com dissecção do pâncreas com isolamento dos vasos esplênicos. Ressecção do baço devido à infilttração tumoral de hilo esplênico. Secção da cauda do pâncreas com sutura mecânica.

A análise histopatológica da peça demonstrou volumosa massa tumoral de superfície castanha com áreas vinhosas, aspecto rugoso e brilhante com aderências filamentares. Aos cortes, tecido tumoral avermelhado com áreas esbranquiçadas, aspecto amolecido, consistência macia e friável. Microscopicamente em cortes corados por hematoxilina eosina, o pâncreas apresentava neoplasia com extensa necrose e hemorragia. Nas inúmeras amostras realizadas havia raras estruturas papilares compostas por células uniformes com núcleos regulares, por vezes formando lençóis. Presença de cápsula fibrosa. Aspecto histológico fortemente sugestivo de neoplasia pseudopapilar sólida de pâncreas. A análise do baço não demonstrou infiltrações neoplásicas.

A paciente apresentou boa evolução clínica, recebendo alta hospitalar cinco dias após a cirurgia.

\section{Discussão}

Tumor sólido pseudopapilar pancreático é um tumor muito raro, com baixo potencial maligno, e ocorre predominantemente $(90 \%)$ em mulheres jovens na segunda ou terceira décadas de vida ${ }^{4,5,6,7}$.

Os autores em geral consideram que características histológicas são necessárias para o diagnóstico. Ozan Karatag e cols. sugerem que, com a visualização de sinais característicos pela ressonância magnética, não há necessidade de biópsia. Em nosso relato de caso, o diagnóstico foi feito por meio de exame histopatológico do tumor. A ressecção do tumor, quando possível, é curativa na maioria dos casos; porém, recidivas locais podem ocorrer. Metástases à distância podem ocorrer em menos de $15 \%$ dos casos, sendo o fígado o principal órgão acometido ${ }^{4,6}$.

A taxa de sobrevida, mesmo após metástase hepática, é alta pela natureza benigna do tumor. Alguns autores correlacionam a incidência de metástases à presença de invasão de estruturas locais pelo tumor ${ }^{4}$.

Nagri S. e cols. demonstraram que o tumor sólido pseudopapilar de pâncreas pode estar presente em pessoas idosas e que metástases após a ressecção primária podem ocorrer sugerindo então acompanhamento subsequente para os pacientes após a cirurgia ${ }^{4}$.

O tumor é um achado casual na maioria das vezes, no entanto dor abdominal é um sinal importante; assim como massa abdominal, anorexia, perda de peso e sintomas relacionados à compressão de órgãos adjacentes podem estar presentes. Os marcadores tumorais geralmente encontram-se normais nestes casos por se tratar de tumor benigno ${ }^{5}$

Macrospcopicamente, os tumores sólidos pseudopapilares do pâncreas geralmente apresentam-se (diâmetro de mediana 10,3 cm), comumente, com extensa área hemorrágica e degenaração pseudocística. Possuem boa delimitação, envoltos por uma cápsula incompleta, e raramente invadem órgãos adjacentes ${ }^{5,7}$.

Apesar de ser um tumor de natureza benigna, Adamthwaite e cols. descreveram dois casos com apresentações tumorais agressivas nos quais haviam componentes histopatológicos atípicos ${ }^{6}$. Estes tumores apresentavam crescimento difuso, extensiva área de necrose, atipia nuclear e taxa 
de mitose elevada. Demonstrando assim que há uma variabilidade na apresentação e clínica do tumor.

A patogênese deste tumor ainda é controversa. Alguns autores defendem uma possível origem endócrina enquanto outros acreditam numa origem de células acinares, ductais ou totipotentes ${ }^{6}$

Adenoma microcístico seroso, neoplasma císitico mucinoso, tumor cístico de ilhotas pancreáticas, pancreaticoblastoma e pseudocisto hemorrágico calcificado são diagnósticos diferenciais quando há uma massa pancreática com conteúdo cístico e sólido. Para o diagnóstico diferencial, o uso de coloração com hematoxilina eosina na análise microscópica e a visualização macroscópica são mais importantes do que a imuno-histoquímica, pois esta não fornece marcadores para o tumor sólido pseudopapilar de pâncreas ${ }^{5,7,8}$.

O diagnóstico de tumor sólido pseupapilar de pâncreas possui quatro pontos importantes que devem ser considerados, como maior incidência em mulheres jovens, tumor abdominal com ou sem dor, ausência de achados hematológicos ou bioquímicos anormais e conteúdo tumoral sólido e cístico com hemorragia e necrose no ultrassom, tomografia computadorizada e ressonância magnética ${ }^{8,9}$. Os achados de exame clínico no caso relatado corroboram com os quatro pontos citados pelos autores.

A ressecção cirúrgica é o tratamento de escolha. Baseia-se na retirada completa do tumor com pancreatectomia distal ou pancreaticoduodenectomia. A ressecção extensa de gânglios linfáticos não é indicada pela maioria dos autores. Na avaliação diagnóstica, o ultrassom abdominal, a tomografia computadorizada, ressonância magnética, biópsia por agulha fina e o congelamento de fragmento tumoral para análise histopatológica intraoperatório são válidos. $\mathrm{Na}$ presença de sintomas clínicos e sinais radiológicos, a aspiração citológica e imuno-histoquímica com ou sem fragmento de biópsia fornecem um diagnóstico preci$\mathrm{so}^{6,8}$. O tratamento realizado para a paciente do caso relatado vai de acordo com o que a literatura consultada preconiza.

\section{Referências}

1- Costa-Neto GD, Amico EC, Costa GID. Tumor sólidocístico pseudopapilar do pâncreas (tumor de Frantz). Estudo de quatro casos. Arq Gastroenterol. 2004; 41(4):259-62.

2- Iribarren Díaz M, Castro Parga G, Fernández Martín R, Meléndez Villar R, Freiria Barreiro G, García Lorenzo F, Gil Gil P. Neoplasia epitelial pseudopapilar sólido-quística de páncreas (tumor de Frantz). Estudio de dos nuevos casos. Rev Esp Enferm Dig. 2004 Dec; 96(12):877-8.

3- Cervantes-Monteil F, Florez-Zorrilla C, Alvarez-Martínez I. Tumor pseudopapilar sólido-quístico de páncreas. Presentación aguda postraumática. Reporte de un caso y revisión de la literatura. Rev Gastroenterol Mex. 2002 AprJun;67(2):93-6.

4- Nagri S, Abdu A, Anand S, Krishnaiah M, Arya V. Liver Metastasis Four Years after Whipple's Resection for SolidPseudopapillary Tumor of the Pancreas. JOP. 2007 Mar 10;8(2):223-7.

5- Karagülle E, Yildirim E, Türk E, Kiyici H, Karakayali H. Solid pseudopapillary tumor of the pancreas: A case report. Turk J Gastroenterol. 2006 Dec;17(4):316-9. 6- Adamthwaite JA, Verbeke CS, Stringer MD, Guillou PJ, Menon KV. Solid Pseudopapillary Tumour of the Pancreas: Diverse Presentation, Outcome and Histology. JOP. 2006 Nov;7(6):635-42.

7- Karatag O, Yenice G, Ozkurt H, Basak M, Basaran C, Yilmaz B. A case of solid pseudopapillary tumor of the pancreas. World J Gastroenterol. 2006 Oct;12(38):623943.

8- Aydiner F, Erinanç H, Sava B, Erden E, Karayalçin K. Solid pseudopapillary tumor of the pancreas: Emphasis on differential diagnosis from aggressive tumors of the pancreas. Turk J Gastroenterol. 2006 Sep;17(3):219-22.

9- Asano T, Seya T, Tanaka N, Ooaki Y, Fujino O. A 13year-old girl with a Preoperatively Diagnosed Solid Cystic Tumor of the Pancreas. J Nippon Med Sch. 2006 Aug;73(4):231-4. 


\section{Endereço para correspondência:}

Av Silva Jardim 2.833, apto. 501

Água Verde, Curitiba - Paraná

CEP: 80240-020

\section{Endereço Eletrônico:}

ivanmalufjr@yahoo.com.br 